\title{
Indicadores de Compulsão Alimentar Periódica em Pós-operatório de Cirurgia Bariátrica
}

\author{
Clarissa Nesi Venzon \\ João Carlos Alchieri \\ Universidade Federal do Rio Grande do Norte \\ Natal, RN, Brasil
}

\begin{abstract}
RESUMO
A obesidade mórbida se relaciona a altas taxas de morbidade e mortalidade. A cirurgia bariátrica (CB) tem se mostrado eficiente levando à perda rápida de peso e remissão de comorbidezes. Encontra-se maior incidência de psicopatologias nesta população. A Compulsão Alimentar Periódica (CAP) tem sido associada ao novo ganho de peso pós-cirúrgico. Este estudo investigou indicadores de CAP em 40 pacientes pós-cirúrgicos submetidos à $\mathrm{CB}$ há pelo menos 2 anos, divididos em Grupo 1 e 2, com perda de mais de 50\% do peso inicial excedente e com perda menor de 50\%, respectivamente. O método de Rorschach-SC e a Escala de Compulsão Alimentar Periódica foram os instrumentos utilizados. Os resultados indicaram diferenças significativas com elevações no Grupo 1 de $\operatorname{CAP}(p<0,01)$, presença de impulsividade $(p<0,05)$ e dificuldade de integração perceptiva $(p<0,05)$. Conclui-se que CAP no pós-cirúrgico pode ser considerado como um possível indicador de reganho de peso.
\end{abstract}

Palavras-chave: Obesidade mórbida; ECAP; Rorschach-SC; Bypass Gástrico.

\begin{abstract}
Binge Eating Indicators after Bariatric Surgery

Morbid obesity is related to high rates of morbidity and mortality. Bariatric Surgery has been shown effective, leading to rapid weight loss and remission of comorbidities. Higher incidence of psychopathologies is in this population. Binge Eating has been linked to the new weight gain after surgery. This study investigated indicators on Binge Eating in 40 post-surgical patients undergoing Bariatric Surgery at least 2 years earlier, who were divided into Group 1 and 2, with a loss of more than $50 \%$ of the initial weight loss and less than $50 \%$, respectively. The Rorschach method-SC and the Binge Eating Scale were the instruments used. The results indicated significant differences with elevations in Binge Eating Group $1(p<0.01)$, presence of impulsivity $(p<0.05)$ and difficulty in perceptual integration $(p<0.05)$. It is concluded that Binge Eating after surgery is a precursor of weight regain and should be treated carefully.
\end{abstract}

Keywords: Morbid obesity; ECAP; Rorschach-SC; Gastric Bypass.

\section{RESUMEN}

\section{Indicadores Atracón en Post-quirúrgicos de la Cirugía Bariátrica}

La obesidad mórbida se relaciona con altas tasas de morbilidad y mortalidad. La cirugía bariátrica (CB) se ha mostrado eficaz que conduce a la pérdida de peso rápida y la remisión de las comorbilidades. Es mayor la incidencia de la psicopatología en esta población. El trastorno por atracón se ha relacionado con el nuevo aumento de peso después de la cirugía. Este estudio indicadores investigados en la tapa 40 pacientes post-quirúrgicos sometidos a CB durante al menos 2 años fueron divididos en Grupo 1 y 2, con una pérdida de más del $50 \%$ de la pérdida de peso inicial y más de 50\% menos, respectivamente. El método de Rorschach-SC y la Escala de atracón fueron los instrumentos utilizados. Los resultados indicaron diferencias significativas en las elevaciones de Grupo Trastorno por Atracón 1 $(p<0,01)$, la presencia de la impulsividad $(p<0,05)$ y la dificultad en la integración perceptual $(p<0,05)$. Llegamos a la conclusión de que la trastorno por atracón después de la cirugía es un precursor de la recuperación de peso y debe ser tratado con cuidado.

Palabras clave: Obesidad mórbida; ECAP; Rorschach-SC; Bypass Gástrico. 


\section{OBESIDADE}

A obesidade tornou-se foco de atenção dos pesquisadores nos últimos anos devido ao aumento vertiginoso de indivíduos com sobrepeso e obesidade na população mundial e ao consequente impacto gerado em âmbito individual, familiar, econômico e social (IBGE, 2010; 0MS, 2012). Segundo a Organização Mundial de Saúde [OMS] (2012), o percentual mundial de obesos duplicou no período compreendido entre 1980 e 2008 . No Brasil, dados informados pelo Instituto Brasileiro de Geografia e Estatística [IBGE] (2010) indicam que as taxas de obesidade e sobrepeso triplicaram desde o ano de 1974. A obesidade mórbida acompanhou esta tendência atualmente representando $6,8 \%$ da população mundial (NIH, 2012), e no Brasil, segundo de acordo com a Sociedade Brasileira de Cirurgia Metabólica [SBCBM] (2008) este percentual é de $3 \%$.

O excesso de peso está frequentemente associado a doenças físicas, conhecidas como de origem mecânica (apneia do sono, problemas articulares, Hipertensão Arterial, etc.) ou metabólica (Diabetes tipo II, Aterosclerose, distúrbios hormonais, etc.). Transtornos clínicos psiquiátricos, comportamentais e de personalidade estão com frequência presentes nos quadros de obesidade. Os transtornos clínicos relacionados à Ansiedade, Depressão e comportamento alimentar são os de maior prevalência. Entre os transtornos Alimentares (TAs), os do tipo Transtorno Compulsivo Alimentar Periódico (TCAP) e a Compulsão Alimentar Periódica (CAP) possuem alta incidência principalmente entre os obesos graves. Os transtornos de personalidade variam em incidência de acordo com o grau de obesidade. A união dos fatores físicos e psicológicos resulta em uma diminuição da qualidade de vida do paciente obeso, também proporcional ao grau de obesidade apresentado (Sandberg et al., 2013; Tavares, Nunes \& Santos, 2010). Apesar de ser um consenso que a obesidade sofre influência direta dos aspectos psicológicos, o entendimento desta associação e da relação causal existente é menos claro do que o das doenças físicas associadas à obesidade, por este motivo há necessidade de mais estudos que elucidem essas associações (Luppino, et al., 2010; Rojas, Brante, Miranda, \& Pérez-Luco, 2011).

Segundo o consenso multissocietário (SBCBM, 2010) a obesidade é subdividida em três níveis (I, II e III) relacionados ao Índice de Massa Corporal (IMC) e riscos envolvidos. A pesquisa em questão focaliza a população que apresenta obesidade Grau III, também denominada como obesidade grave, ou mórbida (IMC maior ou igual a $40 \mathrm{~m}^{2} / \mathrm{kg}$ ). Os obesos graves têm índices de mortalidade significativamente maiores que os obesos com graus menores, devido a maior incidência das doenças relacionadas à obesidade, fato que justifica sua denominação, e a necessidade de ações terapêuticas urgentes, com rápida resposta de resultados em relação à perda de peso.

\section{Tratamento da obesidade}

O tratamento tradicional da obesidade envolve a associação de dieta hipocalórica e atividade física e nos casos mais graves associado ao uso de medicação (Tavares, Nunes, \& Santos, 2010). No entanto, apenas medidas comportamentais e farmacológicas não são eficazes na maioria dos casos, principalmente no que tange a manutenção em longo prazo da perda de peso obtida, já que a obesidade é uma doença orgânica de etiologia multifatorial, sofrendo impacto de aspectos psicológicos, orgânicos e sociais (Román, Martínez, Gonzáles, Vicente, \& Rodríguez-Marín, 2012). Nos casos da obesidade mórbida a ineficácia do tratamento tradicional torna-se mais evidente, um dos motivos citados na literatura é a dificuldade que estes pacientes têm de aumentar o gasto calórico através de atividades físicas, já que o excesso de peso dificulta a prática de atividades aeróbicas de alto impacto devido às comorbidades associadas às articulações e dificuldades de mobilidade (Hintze, Bevilaqua, Pimentel, \& Junior, 2011). Resultados de diversas pesquisas revelam conclusões indicativas de que o tratamento da obesidade grave baseado através de mudança de estilo de vida é eficaz em relação à diminuição das doenças relacionadas à obesidade, entre elas: a Diabetes Tipo II (Unick, et al., 2011) e doenças cardiovasculares (Hofso, et al., 2010). Danielsen, Svendsen, Mæhlume SundgotBorgen (2013), verificaram que além dos benefícios cardiovasculares e diminuição glicêmica, a atividade física intensa está significativamente relacionada com perda de peso, saciedade e controle de episódios de comer compulsivo.

Apesar disto, estudos revelam que não há perda de peso significativa entre os obesos graves, apenas com mudanças de estilo de vida, sendo encontradas perdas em torno de 8 a $10 \%$ do peso inicial em um ano de tratamento (Hofso, et al., 2010; Unick, et al., 2011). Danielsen, et al. (2013), em estudo comparativo entre resultados do tratamento cirúrgico e de tratamento baseado em mudanças de estilo de vida, encontraram resultados em relação a percentual de perda de $30 \%$ e $8 \%$ do peso inicial, respectivamente, nas duas modalidades de tratamento, após um ano de intervenção. Porto (2012), destaca que os benefícios do tratamento cirúrgico da obesidade levam à perda de aproximadamente $50 \%$ do peso inicial no primeiro ano 
pós-cirúrgico; após dois anos de cirurgia esta perda pode chegar a 76\% do peso excedente inicial (Zeve, Novais, \& Júnior, 2012). Diversas pesquisas referem que o paciente obeso mórbido obtém maiores benefícios de forma geral através da Cirurgia Bariátrica, ocorrendo a redução do grau de obesidade, diminuição, ou remissão das doenças relacionadas à obesidade e consequente melhora da qualidade de vida (Kubik, Gill, Laffin, \& Karmali, 2013; Toledo, et al., 2010). Danielsen, et al. (2013), salientam que a mudança de estilo de vida é uma alternativa viável de tratamento para obesos graves tendo em vista às melhorias das doenças relacionadas à obesidade, apesar de menor perda de peso, sendo esta uma possibilidade para aqueles que não podem, ou não desejam fazer a cirurgia bariátrica, bem como pelo fato de não ser viável responder plenamente à demanda de toda a população de obesos graves apenas com a cirurgia da obesidade.

Por estes motivos, a Cirurgia Bariátrica vem sendo considerada a opção de escolha para o tratamento da obesidade mórbida, pois promove a redução drástica do consumo calórico e consequentemente obtenção de êxito cirúrgico, isto é, perda de pelo menos $50 \%$ do peso excedente inicial (PEI), considerado um resultado eficaz em relação à perda de peso levando à redução ou até remissão das doenças associadas à obesidade, podendo o paciente perder $46 \%$ do seu peso total inicial nos dois primeiros anos pós-cirúrgico (Zeve, Novais \& Junior, 2012).

O tratamento cirúrgico da obesidade requer atenção e é indicado apenas para obesos graves (IMC $>40$ ), ou obesos com IMC a partir de $35 \mathrm{~kg} / \mathrm{m}^{2}$ que apresentem comorbidades clínicas associadas e tentativas sucessivas e fracassadas de perda de peso com tratamento clínico (SBCM, 2008). No entanto, a indicação do tratamento cirúrgico para obesidade não se restringe ao IMC, riscos comórbidos e ausência de contraindicações clínicas pré-operatórias. Os aspectos psicológicos e de desordens psiquiátricas devem ser avaliados previamente, já que é sabido que estes influenciam no período pós-operatório e nos resultados cirúrgicos, havendo a necessidade de avaliação psicológica prévia à tomada de decisão (Sarwer, Wadder, \& Fabricatore, 2005).

O preparo cirúrgico da cirurgia da obesidade apresenta características peculiares que vão além dos cuidados clínicos dos quais todo o procedimento cirúrgico prescinde, como os exames laboratoriais, por exemplo. $\mathrm{O}$ candidato à cirurgia bariátrica deve ser avaliado e preparado através de consultas psicoeducacionais para lidar com as mudanças físicas, fisiológicas, sociais, psicológicas e comportamentais que advirão da cirurgia, com vistas a obter bons resultados (Wadden \& Sarwer, 2006; Sockalingam, et al., 2012). O período pré-operatório visa atingir dois objetivos principais: avaliar aptidão psicológica para a submissão à cirurgia e preparar o candidato para o período pós-operatório, justificando assim, a obrigatoriedade de profissionais da área da saúde mental na equipe multidisciplinar (Ministério da Saúde, 2013; SBCM, 2010).

Entre as orientações presentes nos consensos de cirurgia bariátrica internacional e nacional estão os acompanhamentos pré e pós operatório com psicólogo ou psiquiatra, no entanto, a forma como estes devem ser feitos não está especificada (SBCM, 2010; Mechanick, et al., 2008). Na prática há uma tendência ser priorizado o período de preparo, sendo acompanhado de forma mais rígida, pois dele depende a efetivação da cirurgia, já o acompanhamento pós-operatório tende a ser baseado em orientações médicas, que nem sempre são executadas pelo paciente, apesar de ser nesta fase que se garantirá a manutenção dos resultados cirúrgicos. Bassan, et al. (2012), em pesquisa feita com 80 pacientes pós-cirúrgicos de Cirurgia Bariátrica, após dois anos de cirurgia, constataram que $23 \%$ da amostra apresentou reganho de peso, os resultados indicaram associação positiva entre aumento de peso e descuido alimentar, não inserção de atividade física na rotina dos pacientes no período pós-operatório e abandono de acompanhamento psicológico. As autoras ainda referem que o tempo de cirurgia e a qualidade da dieta são preditores independentes de reganho de peso, estimulando mais estudos para com o período pós-operatório visando o planejamento terapêutico. Reforçando a ideia de que o acompanhamento póscirúrgico é tão importante quanto o pré-cirúrgico, apesar de ser menos valorizado nos procedimentos das equipes cirúrgicas (Peacock \& Zizzi, 2012).

Apesar de a maioria dos pacientes obesos graves se beneficiarem com a perda de peso através da cirurgia, recentes pesquisas indicam que um percentual entre $20 \%$ a $30 \%$ de casos apresenta incremento de peso após dois anos pós-cirúrgico, sendo considerado fracasso cirúrgico a perda menor que $50 \%$ do $\mathrm{PEI}$ ou o reganho de mais que $10 \%$ do peso perdido com a cirurgia (Bassan, Gomes, Laura, \& Carvalho, 2012; Pataky, Carrard, \& Golay, 2011; Porto, 2012), um percentual maior que $30 \%$ de reganho de peso pós-cirúrgico é referido em estudos feitos após cinco anos de cirurgia (Toledo, Camilo, Guimarães, Moraes, \& Soares, 2010). As causas deste elevado percentual de "fracasso" do tratamento cirúrgico em longo prazo, ainda não são claras, necessitando de mais estudos que envolvam os diferentes aspectos relacionados à obesidade, incluindo os aspectos psicológicos, foco deste estudo. 


\section{Aspectos psicológicos da obesidade mórbida}

A obesidade é uma doença de base orgânica, no entanto, quando se trata do ser humano, o corpo e "psique" fazem parte de um sistema, não se pode pensar em corpo sem relacioná-lo com o psiquismo. A obesidade afeta e é afetada tanto pelo corpo, como pela mente. Na visão psicanalítica, o corpo funciona como um palco onde os conflitos inconscientes encenam seus dramas, e a obesidade, por vezes, é um sintoma de aspectos psicopatológicos geradores de condutas desadaptadas (Lindenmeyer, 2012). Apesar disso, diversos fatores além dos conflitos inconscientes se relacionam à conduta alimentar inadequada levando à obesidade, além dos aspectos sociais e estilo de vida, os aspectos neurobiológicos vêm sendo continuamente estudados e os resultados indicam participação importante dos mesmos neste fenômeno, um exemplo disso é o temperamento que possui base biológica tem se mostrado relacionado aos TAs, que terminam por ter impacto sob a personalidade do indivíduo (Hilbert, Tuschen-Caffier, \& Hartmann, 2011; Quinton, Smith, \& Joiner, 2011; Schulz \& Laessle, 2011).

Não há indicativo científico de um tipo de personalidade ou de transtorno psiquiátrico exclusivo do obeso mórbido, e sim, tipos de funcionamento psicológico que compreendem algumas características comuns a esta população (Marcus \& Wildes, 2012) como: psicopatologias comórbidas, estilo de vida (Wedin, et al., 2012), traços de personalidade e temperamento(Valenti,Omizo, \& Maledrona, 2011; Vervaet, van Heeringen, \& Audenaert, 2004) aspectos relacionados à autoestima (Rojas, et al., 2011; Elfhag, 2010) somados à singularidade de cada indivíduo obeso (Claes, et al., 2006). Apesar de não ser especificada uma dimensão de personalidade única, relacionada à obesidade, diversos estudos trazem diferenças fenotípicas comportamentais entre os tipos de Transtornos Alimentares, entre eles o transtorno de Compulsão Alimentar Periódica (Cassim \& von Hanson, 2005).

Pesquisas indicam que os aspectos psicológicos do obeso pós-cirúrgico possuem relação importante com o fracasso ou êxito cirúrgico. A alta incidência de compulsão alimentar associada à piora do quadro da obesidade e, do pós-operatório da cirurgia nos chama a atenção e justificam maiores estudos a respeito (Machado, Zilbertein, Cecconello, \& Monteiro, 2008). A compulsão, em diferentes intensidades, é um comportamento que está presente em um grande número de casos, podendo ser associada positivamente à obesidade e ao prejuízo dos resultados cirúrgicos (Dahl, et al., 2010; Niego, 2007; Sandberg, et al., 2013).

\section{Compulsão Alimentar Periódica}

A Compulsão Alimentar Periódica (CAP) foi mencionada pela primeira vez na década de 50 , por Stunkard, no entanto, somente em 1994 foi considerada como entidade psicopatológica e incluída no apêndice $\mathrm{B}$ do Manual diagnóstico e estatístico dos Transtornos Mentais (DSM IV). Devido à alta prevalência de TCAP em relação aos outros TAs na população com obesidade em diferentes graus, e as comorbidades psiquiátricas geralmente associadas a ele, o TCAP foi incluído no DSM-5 como um TA especificado. Os critérios diagnósticos de TCAP sofreram alteração em relação à frequência de episódios compulsivos semanais, um episódio semanal; e duração dos sintomas, pelo menos três meses. Episódios de Binge recorrentes, sem o preenchimento de todo os critérios para o TCAP são denominados de Compulsão Alimentar Periódica.

Os Transtornos Alimentares (TAs) como: Bulimia, Comer Noturno e com maior ênfase a Compulsão Alimentar Periódica (CAP) ou Transtorno de Compulsão Alimentar Periódica (TCAP) propriamente dito, são usualmente encontrados entre a população obesa, valendo salientar que CAP também tem alta incidência entre pessoas sem obesidade, com TCAP subclínico (Kelly, Bulik, \& Mazzeo, 2013). A ocorrência de TCAP, CAP (Dahl, et al., 2010) e o Comer Noturno são mais frequentes nos casos de obesidade grave (Niego, 2007; Sandberg, et al., 2013). Em pesquisa feita com 107 obesos graves candidatos à cirurgia da obesidade, foram encontrados apenas $0,6 \%$ de sujeitos diagnosticados com Bulimia Nervosa, 3,8\% com Transtorno Alimentar Sem Outra Especificação, 13,4\% com TCAP e 14,6\% com CAP (Dahl, et al., 2010).

Uma variedade de estudos traz como resultado uma associação positiva entre a compulsão alimentar e obesidade (Borges, Jorge, Morgan, Silveira, \& Custódio, 2002; Vitolo, Bortolini, \& Horta, 2006). No Brasil de $27 \%$ a $47 \%$ dos pacientes que se submetem à cirurgia bariátrica apresentam compulsão alimentar (Machado, Zilbertein, Cecconello, \& Monteiro, 2008).

Em pesquisa feita com mulheres com Transtorno de Compulsão Alimentar Periódica (TCAP), os resultados indicaram que as mulheres da amostra apresentaram tendência a unir perceptos inadequados nas respostas do Rorschach, como também apresentam dificuldade na capacidade de planejamento e organização, sugerindo que isso influencia negativamente na adesão ao tratamento de obesidade (Passos, Yazigi, \& Claudino, 2008). Corroborando com tais resultados, estudos recentes sobre as diferenças fenotípicas entre os TAs evidenciam diferenças de processamento perceptivo entre pacientes com Anorexia Nervosa e Bulimia 
Nervosa com recorrentes Binges, evidenciando tendência de maior dificuldade de integração perceptiva nos casos de Bulimia e nos casos de Anorexia uma maior atenção aos detalhes (Roberts, Tchanturia, \& Treasure, 2012). Treasure e Shimidt (2013) relacionam a maior atenção a detalhes, prejuízo da visão do todo e rigidez cognitiva das anoréxicas às questões de distorção da imagem corporal e sintomatologia obsessiva compulsiva com consequente envolvimento em dietas de forma rígida. Em relação a respostas fisiológicas ao estresse em pacientes com BN e TCAP, Hilbert, et al. (2011) concluíram que estresses relacionados aos relacionamentos interpessoais levam ao comer compulsivo gerando mais sintomas de insegurança nos pacientes com TCAP, enquanto na Bulimia Nervosa geram mais sintomas depressivos. Schulz e Laessle (2012), também encontraram resultados de estressores associados a maior ingestão de alimento, de forma mais rápida e com incapacidade de percepção de saciedade em obesos com TCAP quando comparados com obesos sem TCAP expostos aos mesmos estressores.

Diferenças também são encontradas entre os TAs em relação à autoimagem, embora a distorção de imagem corporal apresente-se em maior evidência relacionada à Anorexia e Bulimia Nervosa, pesquisas encontram evidências de aumento na percepção corporal, somado a desconforto e preocupação com a imagem corporal em pacientes obesas com TCAP do que sem (Ahrberg, Trojca, Nasrawi, \& Vocks, 2011; Nicoli \& Junior, 2011).

Independente de qual a origem da compulsão alimentar o fato é que ela é um complicador no pósoperatório da cirurgia bariátrica, devendo ser bem avaliada no pré-operatório, e quando detectada o planejamento terapêutico pré e pós-cirúrgico deve ser feito visando focar o seu tratamento para que melhores resultados pós-cirúrgicos sejam alcançados e mantidos (Oliveira \& Yoshida, 2009). Machado (2008), em seu estudo não verificou mudanças entre o pré e o pósoperatório em relação à diminuição da compulsão alimentar, indicando alguns casos de aumento na mesma, o que a torna um problema.

Este estudo teve como objetivo geral a investigação de indicadores de CAP em pacientes que foram submetidos à Cirurgia Bariátrica há pelo menos dois anos. Tendo como objetivos específicos verificar a incidência de CAP e possíveis diferenças de funcionamento de personalidade e temperamento entre indivíduos com e sem CAP, através do método de Rorschach - Sistema Compreensivo.

\section{Aspectos Éticos da Pesquisa}

A pesquisa está de acordo com os procedimentos éticos exigidos para estudos envolvendo seres humanos ou animais, nos termos das Resoluções 196/96 e 251/97 do Conselho Nacional de Saúde, foi submetida à aprovação e obteve anuência do Comitê de Ética da Universidade Federal do Rio Grande do Norte, sob protocolo 0319.0.51.000-11, na data de 25 de abril de 2011.

\section{MÉTODO}

\section{Participantes}

Este estudo foi feito no Hospital Universitário Onofre Lopes, situado na cidade de Natal-RN. A população envolvida foi de pacientes submetidos à Cirurgia Bariátrica do tipo Bypass Gástrico há pelo menos dois anos, no Serviço de Cirurgia da Obesidade e Doenças Relacionadas à Obesidade (SCODE) do referido Hospital. O cálculo amostral foi baseado em levantamento do número de cirurgias feitas entre o ano de 2006, período em que iniciaram as cirurgias bariátricas no SCODE, a abril de 2011, período que contemplava os dois anos mínimos de período pós-cirúrgico. Foram encontrados registros de 156 cirurgias feitas e aproximadamente 26 casos de novo ganho de peso. Contudo, os dados não são precisos devido a alguns prontuários não estarem completos. A partir destes números definiu-se que a amostra seria composta pelos 26 casos de novo ganho de peso e 26 pacientes que obtiveram êxito cirúrgico.

Os critérios de inclusão utilizados foram participantes acima de 21 anos, de ambos os sexos, alfabetizados, que já haviam sido submetidos à cirurgia Bariátrica do tipo Bypass Gástrico há pelo menos dois anos, com IMC $>40$ antes da cirurgia, sem prejuízo cognitivo grave ou doença psiquiátrica, ou problemas acuidade visual não corrigida por lentes que fossem impeditivos à administração dos instrumentos e que consentiram em participar da pesquisa.

Devido a dificuldades relacionadas à atualização de prontuários e desligamentos dos pacientes do SCODE, morte e mudanças de cidade, bem como não consentimento em participar da pesquisa o número amostral inicial não foi atingido.

Ao fim contou-se com 40 participantes, divididos igualmente em dois grupos a priori; Grupo 1 composto pelos participantes que mantiveram a perda de mais de $50 \%$ do peso excedente inicial (PEI), considerado sucesso cirúrgico e Grupo 2 de pacientes que perderam menos 50\% do PEI, denominado de fracasso cirúrgico.

\section{Instrumentos}

Para a execução da coleta de dados relacionados a este estudo foi utilizado um protocolo de pesquisa constituído por técnicas psicológicas do tipo projetiva 
(Método de Rorschach) e objetiva de autorrelato, Escala de Compulsão Alimentar Periódica (ECAP), como também um questionário sociodemográfico.

\section{Método de Rorschach}

O método de Rorschach é extensamente utilizado nacional e internacionalmente, tanto para a prática clínica, como para pesquisas, sua administração é individual, não havendo restrições do seu uso quanto à alfabetização e grau de escolaridade ainda que sejam verificados diferentes padrões de respostas em nível econômico e de escolaridade (Nascimento, 2010). Importante verificar a presença de prejuízo grave da acuidade visual não corrigida com o uso de lentes, bem como alterações na percepção visual das cores, pois sendo o método baseado na visualização de machas cromáticas e acromáticas estes fatores prejudicam a validade dos resultados. Nesta pesquisa nenhum caso foi detectado.

O método é composto por 10 lâminas (cartões) com manchas originais produzidas por Hermann Rorschach em 1921, (três acromáticas, duas pretas e vermelhas e cinco policromáticas). A tarefa do avaliado é falar com o que as manchas se parecem para ele. Através da classificação de suas respostas são verificados aspectos do funcionamento global e psicodinâmicos da personalidade.

\section{Escala de Compulsão Alimentar Periódica (ECAP)}

A ECAP é uma versão traduzida e adaptada para o Brasil por Freitas e colaboradores em 2001, da escala original Binge Eating Scale (BES) desenvolvida por Gormally, Black, Daston e Rardin, 1982. Consta de um questionário autoaplicável que avalia e quantifica a gravidade das tendências à compulsão alimentar periódica em obesos. Trata-se de uma escala likert de 4 pontos ( 0 a 3), onde 0 representa a ausência de compulsão e 3 o nível mais extremo, é composta por 16 itens que avaliam às cognições, sentimentos e comportamentos relacionados ao episódio de compulsão alimentar (Freitas, 2006). Os escores brutos são classificados conforme orientação dos autores responsáveis pela tradução e adaptação do instrumento, da seguinte forma: ausência de compulsão alimentar, quando os escores são menores ou iguais a 17; compulsão alimentar moderada, escores de 18 a 26; e compulsão alimentar grave, acima de 27 , de acordo com os estudos de validação de Freitas, et al. (2001).

A ECAP apresenta poder discriminatório entre indivíduos obesos com e sem TCAP, no entanto não avalia com precisão a presença de TCAP, pois não possui itens na sua composição que definam a frequência semanal de no mínimo dois Binges semanais, por pelo menos seis meses, sendo estes critérios diagnósticos do TCAP segundo DSM-IV, ainda atual, quando o instrumento foi traduzido. Apesar disso a ECAP avalia com precisão a presença de CAP e dos aspectos cognitivos, comportamentais e psicológicos que envolvem a compulsão alimentar, com indicadores de validade para dimensionar graus de CAP.

\section{Procedimentos}

A pesquisa foi aprovada pelo Comitê de ética da UFRN sob o número 0319.0.051.000-11 na data 25 de abril de 2011. O processo de pesquisa foi iniciado pela análise no banco de dados do SCODE para efetuar o cálculo amostral.

Após a anuência do CEP os participantes em potencial foram convidados a participar da pesquisa e foi marcado o primeiro encontro presencial. No primeiro encontro com cada participante, o teor da pesquisa foi explicado e após a leitura do Termo de Consentimento Livre e Esclarecido com coleta das devidas assinaturas deu-se o início da administração dos instrumentos elencados. Os encontros ocorreram em consultórios do SCODE, do setor de psicologia do HUOL e no consultório particular da pesquisadora.

O primeiro instrumento utilizado foi o questionário sociodemográfico. Logo após ocorreu a administração do Rorschach, feita exclusivamente pela pesquisadora, bem como a posterior classificação das respostas. Optou-se por esta modalidade para neutralizar possíveis divergências na forma de administração e classificação. A ECAP foi administrada pela pesquisadora e alunos voluntários da graduação em psicologia devidamente treinados. A administração do protocolo de pesquisa se deu em um, ou no máximo dois encontros dependendo da disponibilidade de tempo do participante.

Através de tabela de números aleatórios, foram selecionados quatro protocolos, representando $10 \%$ do total de protocolos, que foram analisados por três juízes, peritos no sistema compreensivo, sem terem conhecimento da classificação prévia, chegou-se a $89,14 \%$ de concordância. Os únicos dados que lhes foram oferecidos foram escolaridade e faixa etária.

\section{RESULTADOS}

Os resultados do método de Rorschach-SC, ECAP e questionário, foram armazenados em planilha eletrônica e trabalhados através de análises de estatísticas descritivas e inferenciais, de testes estatísticos não paramétricos, U-Mann Withney e K-Kruskal-Wallis com o auxílio do software Statistical Package for the Social Sciences (SPSS20). 
Foram avaliados 40 pacientes pós-operatórios de cirurgia bariátrica, subdivididos em 2 grupos definidos a priori a partir do critério de resultado cirúrgico; Grupo 1, composto por 20 participantes que obtiveram perda de peso menor do que $50 \%$ do peso excedente inicial (PEI) e Grupo 2, composto por participantes com perda superior a $50 \%$ do PEI.

A amostra contou com uma predominância de participantes do sexo feminino (75\%) com escolaridade em nível Médio (55\%), exercendo atividade remunerada (70\%), em convívio marital (62,5\%), com idade média de 42,2 anos e desvio padrão de 10,5 anos, a renda familiar ficou concentrada na faixa entre 3 a 8 salários mínimos $(60 \%)$.

Os resultados da ECAP indicam que $32,5 \%$ da amostra apresentam indicadores de CAP. Sendo que no Grupo 1, encontrou-se 95\% e no Grupo 2 40\% de CAP. Quando os resultados foram comparados entre os dois grupos, apresentaram-se elevados no grupo 1, que apresentou novo ganho de peso, com diferença significativa do ponto de vista estatístico ( $\mathrm{U}=88,000$, $\mathrm{W}=298,00, p<0.01$ ), a qual descreve também os percentuais de CAP encontrados nos dois grupos.

Em relação à gravidade de CAP encontrada nos dois grupos, salienta-se que não há presença de CAP grave no Grupo 2, e que a CAP moderada se resume a 1 caso, representando 5\% deste grupo. No Grupo 1 foram encontrados 8 casos sem CAP representando $40 \%$ do grupo, enquanto os $60 \%$ restantes se distribuíram entre $40 \%$ de CAP moderado e $20 \%$ de CAP Grave.

As variáveis do Rorschach-SC foram comparadas em relação a presença e ausência de CAP (U-Mann Withney), os resultados indicaram diferença significativa com elevação de mediana entre os participantes com CAP, do determinante Forma Dimensão (FD) $[\mathrm{U}=111,500 \mathrm{~W}=22,500 \mathrm{com}$ $p<0,05]$; e das proporções entre indicativas de falta de controle cognitivo ao lidar com aspectos afetivos $(\mathrm{FC}<\mathrm{CF}+\mathrm{C})[\mathrm{U}=119,000, \mathrm{~W}=210,000$ com $p<0,05)$ e indicativa de prejuízo nas relações interpessoais, com pouca tendência à cooperatividade $(\mathrm{COP}<\mathrm{AG})$ $[\mathrm{U}=141,500, \mathrm{~W}=519,500 \operatorname{com} p<0,05]$.

Quando as variáveis do Rorschach foram comparadas à gravidade de CAP (moderada e grave), nos casos de presença de CAP, os resultados indicaram que as variáveis relacionadas à distorção perceptiva (Sum 6 e M-); imaturidade ao lidar com os impulsos (FM), prejuízo na percepção do outro e de si ( $\mathrm{PHR}>\mathrm{GHR}$ ) estavam aumentados nos casos de CAP grave. Apesar disso encontrou-se busca de relacionamentos baseados na cooperação ( $\mathrm{COP}>\mathrm{AG})$. Enquanto a atenção aos Detalhes $(\mathrm{Dd})$ e conteúdo vestuário $(\mathrm{Cg})$ estão diminuídas de forma significativa em relação aos indicadores de CAP moderada, como pode ser visto na Tabela 1 .

TABELA 1

Variáveis Rorschach e Níveis Gravidade de CAP

\begin{tabular}{|c|c|c|c|c|c|}
\hline & $C A P$ & Medianas & $U^{\mathrm{a}}$ & $W^{b}$ & $p$ \\
\hline \multirow{2}{*}{ FM } & Moderada & 5,67 & \multirow{2}{*}{6,000} & \multirow{2}{*}{51,000} & \multirow{2}{*}{$0,05^{*}$} \\
\hline & Grave & 10,00 & & & \\
\hline \multirow{2}{*}{ Mp FQ- } & Moderada & 5,72 & \multirow{2}{*}{6,500} & \multirow{2}{*}{51,500} & \multirow{2}{*}{$0,03 *$} \\
\hline & Grave & 9,88 & & & \\
\hline \multirow{2}{*}{ GHR } & Moderada & 8,50 & \multirow{2}{*}{4,500} & \multirow{2}{*}{14,500} & \multirow{2}{*}{$0,03 *$} \\
\hline & Grave & 3,63 & & & \\
\hline \multirow{2}{*}{ GHR $<$ PHR } & Moderada & 5,72 & \multirow{2}{*}{6,500} & \multirow{2}{*}{51,500} & \multirow{2}{*}{$0,03 *$} \\
\hline & Grave & 9,88 & & & \\
\hline \multirow{2}{*}{$\mathrm{COP}>\mathrm{AG}$} & Moderada & 5,72 & \multirow{2}{*}{6,500} & \multirow{2}{*}{51,500} & \multirow{2}{*}{$0,03 *$} \\
\hline & Grave & 9,88 & & & \\
\hline \multirow{2}{*}{ Sum6 } & Moderada & 5,44 & \multirow{2}{*}{4,000} & \multirow{2}{*}{49,000} & \multirow{2}{*}{$0,03 *$} \\
\hline & Grave & 10,50 & & & \\
\hline \multirow{2}{*}{ Dd } & Moderada & 8,44 & \multirow{2}{*}{5,000} & \multirow{2}{*}{15,000} & \multirow{2}{*}{$0,04 *$} \\
\hline & Grave & 3,55 & & & \\
\hline \multirow{2}{*}{ Blends/R } & Moderada & 5,50 & \multirow{2}{*}{4,500} & \multirow{2}{*}{49,500} & \multirow{2}{*}{$0,04 *$} \\
\hline & Grave & 10,38 & & & \\
\hline \multirow{2}{*}{$\mathrm{Cg}$} & Moderada & 8,33 & \multirow{2}{*}{6,000} & \multirow{2}{*}{16,000} & \multirow{2}{*}{$0,04 *$} \\
\hline & Grave & 4,00 & & & \\
\hline
\end{tabular}

Fonte: Dados de Pesquisa, 2013.

a) U-Mann-Withney; b) Wilcoxon

$* p<0,01 ; * * p<0,05$.

\section{DISCUSSÃO DOS RESULTADOS}

A distribuição encontrada em relação à idade, sexo e estado civil está de acordo com o que se encontra na literatura referente à população pós-bariátrica, havendo um maior número de cirurgias feitas por mulheres, indivíduos casados e sendo uma população com ampla variação etária (Langaro, Poggere, \& Trentini, 2011).

A incidência da presença de indicadores de Compulsão Alimentar na amostra de 32,5\% corrobora valores de outras pesquisas (Machado, Zilbertein, Cecconello, \& Monteiro, 2008). E o fato de sua presença não se restringir ao Grupo 1 de novo ganho de peso também já foi evidenciada em outros estudos que referem que a compulsão alimentar periódica também está presente na população não obesa (Kelly, Bulik, $\&$ Mazzeo, 2013). A diferença significativa $(p<0,01)$ encontrada entre os dois grupos indicando aumento de CAP no grupo com aumento de peso vai ao encontro de diversas pesquisas que associam CAP a aumento de peso no pós-operatório (Dahl, et al., 2010; Niego, et al., 2007). Não podemos afirmar se o nível de CAP era diferente no período pré-operatório devido ao delineamento transversal da pesquisa, mas concluímos 
que a presença de CAP no pós-operatório pode ser um indicador de ganho de peso pós-cirúrgico. Não foi encontrada diferença significativa entre os grupos 1 e 2 em relação à gravidade de CAP (moderada e grave). Isso se justifica pelo fato de que $100 \%$ dos participantes com CAP Grave se encontrava no Grupo 1 e a maioria dos casos moderados estarem no mesmo grupo, o que reforça a ideia de associação entre indicadores de compulsão alimentar e aumento de peso pós-cirúrgico.

As variáveis do Rorschach que se mostraram significativamente diferentes entre os indivíduos com resultados positivos e negativos na Escala de Compulsão Periódica indicam que o grupo com CAP tem menos controle cognitivo sobre as experiências afetivas, tendendo à impulsividade e perda de controle, já que possuem em maior número respostas de cor sem forma (C) ou com predominância de cor sobre a forma (CF) aspectos indicativos de pouco controle na expressão e vivências afetivas (Nascimento, 2010). Somado a isso foi encontrada uma forma imatura de lidar com as demandas instintivas, reforçando a tendência à impulsividade. Estes resultados corroboram achados de pesquisas anteriores com o Rorschach (Elfhag, 2011; Passos, Yazigi, \& Claudino, 2009). Não foram encontradas diferenças significativas entre os grupos em relação ao indicador de capacidade de controle e tolerância ao Estresse (nota D e Nota D ajustada), outro estudo já havia chegado a estes resultados (Elfhag, 2011). Pode-se pensar que a ocorrência de tais resultados se justifique pelo fato de a compulsão alimentar estar mais associada ao descontrole crônico associado à impulsividade (respostas de cor) como uma forma reativa habitual, do que ao descontrole relacionado a respostas de demandas diárias somadas a estressores externos, com os quais as notas D e D ajustadas se relacionam. Em relação a isso podemos pensar nos estudos que associam Compulsão Alimentar a temperamentos explosivos, e indivíduos impulsivos como encontrado em outras pesquisas (Valenti, Omizo, \& Maledrona, 2011; Vervaet, van Heeringen, \& Audenaert, 2004). A CAP vem sendo associada a problemas nos relacionamentos e autoestima denegrida (Ahrberg, Trojca, Nasrawi, \& Vocks, 2011; Nicoli \& Junior, 2011; Niego, 2007). Os resultados encontrados podem se relacionar a isso devido ao aumento do determinante Forma Dimensão (FD) nos participantes com CAP sugerindo uma tendência a aumento de atividade autoavaliativa que, quando em excesso, somado a distorções perceptivas pode se tornar distorcida, desconfortável prejudicando a autoestima (Exner \& Sendin, 1999). Neste estudo foi verificada relação entre presença de indicadores de CAP e aumento de respostas de movimento agressivo (AG) em relação às respostas de movimento cooperativo (COP), as quais segundo Nascimento (2010) revelam tendência a envolver-se nas relações interpessoais com condutas agressivas e /ou pouco cooperativas, denotando prejuízo nas relações interpessoais. Este aspecto corrobora conclusões de estudo que associa os episódios de compulsão alimentar a estressores emocionais advindos das relações interpessoais (Schulz \& Laessler, 2011).

Importante salientar aqui a relação entre indicadores de desorganização do pensamento e distorções perceptivas identificadas no Rorschach-SC (M-, Sum 6) que está aumentada entre os participantes com indicadores de CAP grave. Tais resultados reforçam a ideia de que não só a compulsão alimentar, mas sua gravidade se relacionam a maiores prejuízos perceptivos como encontrado em outras pesquisas com Rorschach relacionado à CAP (Passos, Yazigi, \& Claudino, 2008; Elfagh, 2011). A elevação destas variáveis podem justificar o aumento da complexidade cognitiva (Blends/R) envolvida no processamento das informações, que se associada às variáveis Sum $6 \mathrm{e}$ M- indicam que tal complexidade não está levando à eficácia na tomada de decisão, sendo fonte de respostas e condutas desadaptadas em relação às demandas diárias. Tais ideias reforçam estudos recentes que relacionam a compulsão alimentar a dificuldades de integração perceptiva e capacidade de planejamento em situações que envolvem os afetos (Colier \& Treasure, 2004; Treasure \& Schimidt, 2013). Ainda acerca dos resultados do Rorschach relacionados à gravidade de indicadores de CAP, pode-se pensar que a presença de conteúdo vestuário $(\mathrm{Cg})$ e Detalhe Incomum $(\mathrm{Dd})$ relacionada à gravidade moderada, leva à reflexão de que estes elementos fazem parte das variáveis que compõem o índice de Hipervigilância (HVI), que se relaciona a atitudes e ideação paranoides e condutas de maior controle, podendo ter efeito atenuante sobre a CAP.

Os resultados reforçam a ideia da importância do período pós-operatório ser acompanhado a longo prazo, de forma mais constante, da que vem acontecendo, já que é reconhecida na literatura uma maior atenção ao período pré-operatório, o qual é válido para planejamento terapêutico, mas não é suficiente para a manutenção dos resultados cirúrgicos. Os dados corroboram com estudos anteriores revelando que esta população pode apresentar características psicológicas singulares como; presença de TCAP, impulsividade, autoestima e autoimagem denegridas (Valenti, Omizo, \& Maledrona, 2011; Vervaet, van Heeringen, \& Audenaert, 2004), autocrítica excessiva, dificuldades nas relações interpessoais (Hilbert et al., 2011), 
distorções perceptivas (Passos, Yazigi, \& Claudino, 2008; Elfagh, 2011) sintomatologia depressiva e ansiosa que exigem atenção e acompanhamento póscirúrgico. Evidenciando que a presença de TCAP e impulsividade no período pós-operatório se relaciona positivamente com novo ganho de peso (Dahl et al., 2010; Niego, et al., 2007), e por este motivo devem ser tratadas em longo prazo. O tratamento deve ser contínuo, e os aspectos psicológicos pertinentes ao período pós-operatório não devem ser negligenciados, pois a cirurgia é apenas uma parte do tratamento da obesidade mórbida.

Após a cirurgia o tratamento deve ser continuado, pois a problemática do possível novo ganho de peso no período pós-operatório continua sendo a mesma da origem da obesidade, desequilíbrio entre ingestão calórica e gasto energético, o que reforça duas importantes ideias: de que a cirurgia não é a solução da obesidade, e sim uma etapa do tratamento que apenas tem êxito quando mudanças de hábitos e comportamentos são efetuadas, devendo a obesidade continuar sendo tratada continuamente; e que aspectos psicológicos avaliados no período anterior à cirurgia embasam o planejamento de tratamento pós-operatório, verificando vulnerabilidades e forças do paciente (van Hout, Boekestein, Fortuin, Pelle, \& van Heck, 2006).

\section{CONCLUSÕES}

Através da análise dos resultados pode-se concluir que há alta incidência de Compulsão Alimentar periódica entre os obesos pós-cirúrgicos da amostra. Sobre os indicadores de gravidade de Compulsão Alimentar Periódica, pode-se dizer que estão relacionados ao aumento de distorções perceptivas e desorganização do pensamento, associada a maior dificuldade em lidar com as demandas instintivas com consequente impulsividade e aumento de imaturidade. Em relação à gravidade de CAP moderada estão relacionados indicadores de presença de maior preocupação com os outros, relativas a ideias de desconfiança e controle. Conclui-se que a presença de indicadores de Compulsão Alimentar periódica no período pós-cirúrgico se relaciona com novo ganho de peso após a cirurgia.

Por fim, este estudo chama a atenção para o período pós-operatório como fase importante do tratamento da obesidade grave. Reforçando ideias de que a cirurgia bariátrica é apenas uma fase do tratamento da obesidade grave, e que a manutenção da perda de peso após a mesma, depende de adequada avaliação psicológica pré-cirúrgica que possibilite adequado planejamento terapêutico em longo prazo.
O tratamento é contínuo, já que a obesidade mórbida é uma doença crônica, envolvendo mudança de hábitos alimentares e comportamentais e de acompanhamento psicológico que abarque fatores psicológicos previstos na fase pré-operatória e àqueles inerentes ao período pós-operatório, respeitando a singularidades de cada paciente.

\section{REFERÊNCIAS}

Ahrberg, M. Trojca, D., Nasrawi, N., \& Vocks, S. (2011). Body image disturbance in binge eating disorder: A Review. European Eating Disorders Review. Recuperado em http://journals. ohiolink.edu/ejc/article.cgi issn=10724133\&issue =v19i0005\& article $=375$ bidibedar

American Psychiatric Association (2002). DSM IV-TR: Manual diagnóstico e estatístico dos transtornos mentais. Porto Alegre: Artes Médicas.

Azevedo, A. P., Santos, C. C., \& Fonseca, D. C. (2004). Transtorno da compulsão alimentar periódica. Psiquiatria Clínica, 31(4), 170-172.

Bassan, F. L., Gomes, D. L., Laura, \& Carvalho, K. M. B. (2012). Comportamento alimentar e reganho de peso após 24 meses de cirurgia bariátrica. Nutrire, 37(Supl.), 36-36. Recuperado em http://www.revistanutrire.org.br/articles/view/ id/4fd72b641 efl faac76000006.

Borges, M. B., Jorge M. R., Morgan C. M., Silveira D. X., \& Custódio. (2002). The binge-eating disorder in Brazilian women on a weight-loss program. Obesity, 10(11), 1127-1134. doi: 10.1038/oby.2002.153

Claes, L., Vandereycken, W., Luyten, P., Soenens, B., Pieters, G., \& Vertommen, H. (2006). Personality prototypes in eating disorders based on the Big Five model. J Pers Disord, 20(4), 401-416. Recuperado em http://guilfordjournals.com/doi/ abs/10.1521/pedi.2006.20.4.401

Collier, D.A., \& Treasure, J.L. (2004). The aetiology of eating disorders. British journal of psychiatry, 185, 363-365. Recuperado em http://bjp.rcpsych.org/content/185/5/363.full.pdf + html

Dahl, J. K., Eriksen, L., Vedul-Kjelsas, E., Strommen, M., Kulseng B., Marvik R., \& Holen, A. (2010). Prevalence of all relevant eating disorders in patients waiting for bariatric surgery: a comparison between patients with and without eating disorders. Eat Weight Disord, 15(4), 247-755. Recuperado em http://www. ncbi.nlm.nih.gov/pubmed/21406948

Danielsen, K. K., Svendsen, M., Mæhlum, S., \& Sundgot-Borgen, J. (2013). Changes in Body Composition, Cardiovascular Disease Risk Factors, and Eating Behavior after an Intensive Lifestyle Intervention with High Volume of Physical Activity in Severely Obese Subjects: A Prospective Clinical Controlled Trial. Journal of Obesity, 2013. Recuperado em http://dx.doi. org/10.1155/2013/325464.

Elfhag, K. (2010). A Synthesis of recent research on obesity with the rorschach and suggestions for the future.Rorschachiana, 31(2), 117-142. doi:10.1027/1192-5604/a000008

Exner Jr., J. E., \& Sendín, C. (1999). Manual de Interpretação do Rorschach para o sistema compreensivo. São Paulo: Casa do Psicólogo.

Freitas, S. R., Lopes, C. S., Coutinho, W., \& Apollinario, J. C. (2001). Tradução e adaptação para o português da Escala de Compulsão Alimentar Periódica. Revista Brasileira de Psiquiatria, 23(4), 215-220. Recuperado em http://www.scielo. br/pdf/\%0D/rbp/v23n4/7169.pdf 
Freitas, S. R. (2006). Instrumentos para avaliação dos transtornos alimentares. In: M. A., Nunes, J. C., Appolinário, A. L., Galvão, \& W. Coutinho (Org.). Transtornos Alimentares e Obesidade. Porto Alegre: Artes Médicas.

Hilbert, A. V. C., Tuschen-Caffier, B., \& Hartmann, A. S. (2011). Psychophysiological responses to idiosyncratic stress in bulimia nervosa and binge eating disorder. Physiology \& Behavior, 104 (5), 770-777. Recuperado em http://dx.doi.org/10.1016/j. physbeh.2011.07.013

Hintze, L. J., Bevilaqua, C. A., Pimentel, E. B., \& Junior, N. N. (2011). Cirurgia bariátrica no Brasil: Bariatric surgery in Brazil. Rev. Ciênc. Méd., 20(3-4), 87-98.

Hofso, D. et al. (2010, Aug 26). Obesity-related cardiovascular risk factors after weight loss: a clinical trial comparing gastric by-pass surgery and intensive lifestyle intervention. Eur J Endocrinol. Recuperado em http://www.eje-online.org/content/163/5/735. short . doi: 10.1530/EJE-10-0514

Instituto Brasileiro de Geografia e Estatística. (2010). Pesquisa de orçamentos familiares 2008-2009: Antropometria e estado nutricional de crianças, adolescentes e adultos no Brasil. Recuperado em http://www.ibge.gov.br/home/presidencia/ noticias/imprensa/ppts/0000000108.pdf

Kelly N. R., Cynthia M. Bulik C. M., \& Mazzeo S. E., (2013). Executive functioning and behavioral impulsivity of young women who binge eat. International Journal of Eating Disorders, 2(46), 127-139.

Langaro, F., Vieira, A. P. K., Poggere, L. C., \& Trentini, C. M. (2011). Características de personalidade de mulheres que se submeteram à cirurgia bariátrica. Avaliação Psicológica, 10(1), 71-79. Recuperado em http://pepsic.bvsalud.org/scielo. php?pid=1677-4712011000100008\&script $=$ sci_arttext

Lindenmeyer, C. 2012 Qual é o estatuto da Psicanálise? Tempo Psicanalítico, 44(2), 341-359. ISSN 0101-4838

Luppino, F. S., et al., (2010). Overweight, Obesity, and Depression: A Systematic Review and Meta-analysis of Longitudinal Studies. Arch Gen Psychiatry, 67(3), 220-229. doi:10.1001/ archgenpsychiatry.2010.2

Machado, C. E. (2008). Estudos sobre a compulsão alimentar em pacientes submetidos à cirurgia bariátrica. Dissertação de Mestrado, Universidade de São Paulo, Brasil.

Machado, C. E., Zilbertein, B., Cecconello, I., \& Monteiro. M. (2008). Compulsão alimentar antes e após a cirurgia bariátrica. ABCD Arquivos Brasileiros de Cirurgia Digestiva, 21(4), 185191. Recuperado em http://www.scielo.br/pdf/abcd/v21n4/ v21n4a07.pdf.

Marcus, M. D., \& Wildes, J. E. (2012). Obesity in DSM-5, Psychiatric Annals, 42(11), 431-435. Recuperado em http:/ www.healio.com/psychiatry/journals/PsycAnn/\%7BF259FB28070B-4D6F-B2B5-D8A46DEFE045\%7D/Obesity-in-emDSM5em. doi: 10.3928/00485713-20121105-10

Mechanick, J. I., Kushner, R. F., Sugerman, H. J., \& Dixon, J. (2008). American Association of Clinical Endocrinologists, The Obesity Society, and American Society for Metabolic \& Bariatric Surgery Medical Guidelines for Clinical Practice for the Perioperative Nutritional, Metabolic, and Nonsurgical Support of the Bariatric Surgery Patient. Surgery of obesity and related diseases, 4(5), 109-184.

Melo, M. M. O. (2011). Compulsão alimentar, imagem corporal e qualidade de vida em crianças e adolescentes obesos. (Dissertação de mestrado), Universidade Federal de Minas Gerais, Belo Horizonte.

Nascimento, R. S. G. F. (2010). Sistema Compreensivo do Rorschach - Teoria, pesquisa e normas para a população brasileira. São Paulo: Casa do Psicólogo.
Nicoli, M. G., \& Junior, R. D. R. L. (2011). Transtorno da compulsão alimentar e percepção da imagem corporal entre estudantes universitários. Comportamentos alimentares, 12(4), 284-288.

Niego, S. (2007). Binge eating in the bariatric surgery population: A review of the literature. (Clinical report). The international journal of eating disorders, 40(4), 349-359.

Oliveira, J. H. A., \& Yoshida, E. M. P. (2009). Avaliação Psicológica de Obesos Grau III Antes e Depois de Cirurgia Bariátrica. Psicologia: Reflexão e Crítica, 22(1), 12-19. Recuperado em http://dx.doi.org/10.1590/S0102-79722009000100003

Organização Mundial de Saúde (2012). World Health Organization 2012. Recuperado em http://www.who.int/about/licensing/ copyright_form/en/index.html. ISBN 9789241564441 (NLM classification: WA 900.1).

Passos, T. C. B. M.; Yazigi, L., \& Claudino, A. (2008). Aspectos ideativos no transtorno da compulsão alimentar periódica: estudo com o Rorschach. Psico-USF, 13(1), 69-74. Recuperado em http://www.scielo.br/pdf/pusf/v13n1/v13n1a09.pdf.

Pataky, Z., Carrard, I., \& Golay, A. (2011). Psychological factors and weight loss in bariatric surgery. Current Opinion in Gastroenterology, 27(2), 167-173. doi:10.1097/MOG.0b013e 3283422482

Portaria nº 424/2013 de 19 de Março. Ministério da Saúde (2013). Recuperado em http://bvsms.saude.gov.br/bvs/saudelegis/ gm/2013/prt0424_19_03_2013.htm

Quinton, S. J., Smith, A. R., \& Joiner, T. (2011). The 2nd to 4th digit ratio (2D:4D) and eating disorder diagnosis in women. Personalidade e Diferenças Individuais, 51(4), 402-405. Recuperado em http://dx.doi.org/10.1016/j.paid.2010.07.024.

Rojas, C., Brante, M., Miranda, E., \& Pérez-Luco, R. (2011). Descripción de manifestaciones ansiosas, depresivas y autoconcepto en pacientes obesos mórbidos, sometidos a cirugía bariátrica. Rev Med Chile, 139(5), 571-578. Recuperado em http://dx.doi.org/10.4067/S0034-98872011000500002.

Román, C. J. V. H., Martínez, E. P., Gonzáles, S. T., Vicente, M. M., \& Rodríguez-Márin, J. (2012). Psychological effects of a structured programme for preparing bariatric surgery patients. Ansiedad y Estrés, 18(2-3), 231-239.

Sandberg, R. M., et al. (2013). Health-related quality of life in obese presurgery patients with and without binge eating disorder, and subdiagnostic binge eating disorders. Journal of Obesity In Hindawi Publishing Corporation, 1-7. Recuperado em http:// dx.doi.org/10.1155/2013/878310. doi: 10.1155/2013/878310

Sarwer, D. B., Wadden, T. A., \& Fabricatore, A. N. (2005), Psychosocial and Behavioral Aspects of Bariatric Surgery. Obesity Research, 13, 639-648. doi: 10.1038/oby.2005.71

Schulz, S., \& Laessle, R. G. (2011). Induzida pelo estresse laboratório comportamento alimentar em mulheres obesas com transtorno de compulsão alimentar. Apetite, 58(2), 457-461.

Sociedade Brasileira de Cirurgia Bariátrica e Metabólica (SBCBM). (2008). Pesquisa inédita da SBCBM revela dados alarmantes sobre a obesidade no Brasil. Boletim SBCBM, 23, 4-5. São Paulo: Target Consultoria em Comunicação.

Sociedade Brasileira de Cirurgia Bariátrica e Metabólica (SBCBM). (2010). Consenso Brasileiro Multissocietário em Cirurgia da Obesidade. Recuperado em http:/www.sbcbm.org.br

Sockalingam, S., et al. (2012). Psychiatric predictors of surgery non-completion following suitability assessment for bariatric surgery. Springer Science + Business Media, 23(2), 205-211. doi: 10.1007/s11695-012-0762-5

Tavares, T. B., Nunes S. M., \& Santos M. O. (2010). Obesidade e qualidade de vida: revisão da literatura. Rev Med Minas Gerais, 20(3), 359-366. Recuperado em http://rmmg.medicina.ufmg.br/ index.php/rmmg/article/viewFile/276/260. 
Toledo, C. C, Camilo, G. B., Guimarães, R. L., Moraes, F. R., \& Soares Jr., C. (2010). Qualidade de vida no pós-operatório tardio de pacientes submetidos à cirurgia bariátrica. Revista APS, 13(2), 202-209. Recuperado em http://www.aps.ufjf.br/index. php/aps/article/viewArticle/441.

Treasure, J., \& Schmidt, U. (2013). The cognitive-interpersonal maintenance model of anorexia nervosa revisited: a summary of the evidence for cognitive, socio-emotional and interpersonal predisposing and perpetuating factors. Journal of Eating Disorders, 1(13), 10. Recuperado em.http://www.jeatdisord. com/content $/ 1 / 1 / 13$

Unick, J. L., et al. (2011). Effectiveness of lifestyle interventions for individuals with severe obesity and type 2 diabetes. Diabetes care, 34, 2152-2157. Recuperado em http://care.diabetesjournals. org/content/34/10/2152.short

Valenti, Omizo, \& Maledrona. (2011). Personality and Obese Body Mass Index. In Howard Steele. The New School Psychology Bulletin, 9(1), 60. New York: NSPB.

van Hout, G. C., Boekestein, P., Fortuin, F. A., Pelle, A. J., \& van Heck, G. L. (2006). Psychosocial functioning following bariatric surgery. Obes Surg, 16(6), 787-94. Recuperado em http://link. springer.com/article/10.1381/096089206777346808

Vervaet, M., van Heeringen, C., \& Audenaert, K. (2004). Personality-related characteristics in restricting versus binging and purging eating disordered patients. Comprehensive Psychiatry, 45(1), 37-43.

Vitolo, M. R., Bortolini, G. A., \& Horta, R. L. (2006). Prevalência de compulsão alimentar entre universitárias de diferentes áreas de estudo. Revista Psiquiatria, 28(1), 20-6. Recuperado em http://www.scielo.br/pdf/rprs/v28n1/v28n1a04.pdf.

Wadden, T. A., \& Sarwer, D. B. (2006). Behavioral assessment of candidates for bariatric surgery: a patient-oriented approach. Surgery for Obesity and Related Diseases, 14(3), 53-62. Recuperado em http://onlinelibrary.wiley.com/doi/10.1038/ oby.2006.283/full.

Wedin, S., Morgan, K., LePage, R., Borckart, J. J. (2012). Presurgical Weight Is Associated with Pain, Functional Impairment, and Anxiety among Gastric Bypass Surgery Patients. Pain Research and Treatment, 12. doi:10.1155/2012/412174

Zeve, J. L. D. M., Novais, P. O., \& Júnior, N. D. O. (2012). Técnicas em cirurgia bariátrica: uma revisão da literatura. Ciência \& Saúde, 5(2), 132-140, http://revistaseletronicas.pucrs.br/ojs/ index.php/faenfi/article/viewFile/10966/8206

Autores:

Clarissa Nesi Venzon - Mestre em Psicologia, Universidade Federal do Rio Grande do Norte.

João Carlos Alchieri - Doutor, Universidade Federal do Rio Grande do Norte.

Endereço para correspondência:

Clarissa Nesi Venzon

Universidade Federal do Rio Grande do Norte

Natal, RN, Brasil

E-mail: clavenzon@hotmail.com

Recebido em: 02.04.2013

Aceito em: 29.05.2014 